\title{
Testing the counter model for perceptual identification: Effects of repetition priming and word frequency
}

\author{
ERIC-JAN M. WAGENMAKERS, RENÉ ZEELENBERG, and JEROEN G. W. RAAIJMAKERS \\ University of Amsterdam, Amsterdam, The Netherlands
}

\begin{abstract}
The counter model for perceptual identification (Ratcliff \& McKoon, 1997) differs from alternative views of word recognition in two important ways. First, it assumes that prior study of a word does not result in increased sensitivity but, rather, in bias. Second, the effects of word frequency and prior study are explained by different mechanisms. In the present experiment, study status and word frequency of target and foil were varied independently. Using a forced-choice task, we replicated the bias effect. However, we also found several interactions between frequency and prior study that are in direct conflict with the counter model. Most important, prior study of both alternatives resulted in an attenuation of the frequency effect and an increase in performance for low-frequency targets, but not for highfrequency targets. These findings suggest that the effects of frequency and prior study are not mediated by completely independent mechanisms.
\end{abstract}

The effects of repetition priming and word frequency are among the most often studied phenomena in visual word recognition. The repetition priming effect in perceptual identification refers to the finding that a briefly flashed word is more likely to be correctly identified if that word appears on a previous study list than if it does not. The frequency effect refers to the finding that performance is better for a high-frequency (HF) target than for a low-frequency (LF) target. In many models, a single mechanism is used to model the relatively high performance for words studied previously and for words with a high frequency of occurrence in natural language. For instance, in the logogen model (Morton, 1969) and in the interactive activation model (McClelland \& Rumelhart, 1981), repetition priming and word frequency both act to reduce the distance from the activation level of a word representation to its threshold value (either by lowering the threshold or by increasing the activation level).

Recently, Ratcliff and McKoon (1997) proposed a counter model for repetition priming and word frequency effects that is radically different from earlier accounts. In this paper, we focus on a central assumption of the counter

R.Z. was supported by a grant from the Foundation for Behavioral and Social Sciences of the Netherlands Organization for Scientific Research. We thank Roger Ratcliff and Gail McKoon for providing us with the instructions used in their experiments on perceptual forced choice. We thank Noortje Jansen, Han van der Maas, Sander Nieuwenhuis, Diane Pecher, and Richard Shiffrin for helpful comments on an earlier draft of this paper. We also thank Geertje Hagedoorn, Chris Leuris, Arnout Koornneef, and Diana Schuitemaker for their help in testing subjects and selecting stimulus materials. Correspondence concerning this article should be addressed to E.-J.M. Wagenmakers or R. Zeelenberg, Department of Psychonomics, Roetersstraat 15, 1018 WB Amsterdam, The Netherlands (e-mail: pn_wagenmakers@macmail.psy. uva.nl or pn_zeelenberg@macmail.psy.uva.nl). model. This assumption holds that repetition priming and word frequency are mediated by different mechanisms. Before discussing the rationale of our experiment, we will briefly describe the counter model as it applies to the perceptual identification forced-choice task. In this task, one word (e.g., BOTHER) is briefly flashed, and the subject subsequently has to choose which of two alternatives (e.g., BOTHER or MOTHER) corresponds to the flashed word.

In the counter model, words are represented as counters. At each time step, a unit of information, a count, can be taken by one (and only one) of the counters. The larger the number of counts in a counter, the more evidence there is that the word corresponding to that counter is the word that has been presented. The counter model distinguishes three types of counts: diagnostic counts, nondiagnostic counts, and null counts. Diagnostic counts are counts that correspond to features that are features of only the target and not the foil (e.g., B in BOTHER-MOTHER). Nondiagnostic counts are counts that correspond to features of both the target and the foil (e.g., o in BOTHERMOTHER). Both diagnostic and nondiagnostic counts are determined by the stimulus. Null counts, however, are not determined by the stimulus and represent random noise in the system. The counter model assumes that in the two-alternative forced-choice task, a decision is made when the total number of counts in one counter exceeds that in the other counter by a criterion amount $k$. As will be discussed later, the assumption that the response criterion is relative to other counters is important.

Suppose that the forced choice is between BOTHER and MOTHER and that the subject has perceived only the last nondiscriminating five letters. In this case, all the counts are nondiagnostic and have a probability of .5 of being taken by the counter of either alternative. When the sub- 
ject has seen nothing at all, there are only random or null counts, and these are also assigned to either counter with a probability of .5. However, subjects may also perceive information that discriminates the presented alternatives (e.g., the first letter of the BOTHER-MOTHER pair). Such information, diagnostic counts, is always taken by the target's counter. Thus, the overall probability $p$ of a count going to the counter of the target word is $p=p s+.5(1-$ $p s)$, where $p s$ equals the probability of a target diagnostic count.

Repetition priming is modeled by a change in the probability that a nondiagnostic or null count (i.e., $1-p s$ ) is accumulated by the counter of the target. For the studied alternative, the probability that a nondiagnostic or a null count is taken by its counter increases (in the simulations of Ratcliff \& McKoon, 1997, the probability increased from .50 to .51 , but the magnitude of the increase is actually a parameter of the model). Thus, prior study leads to a theft of nondiscriminative counts by the counter of the studied alternative. Hence, when the choice is between BOTHER and MOTHER, prior study of MOTHER results in an increase in performance when MOTHER is the target word, but it results in a decrease in performance when BOTHER is the target word. This pattern of costs and benefits of prior study is termed bias. In other words, people are biased to "see" the word that has been studied previously, regardless of whether the studied word is the target or the foil. It is further assumed that the counter of a studied word acts as a weak attractor, in the sense that it is only capable of theft from alternatives very similar to it (see Schooler, Shiffrin, \& Raaijmakers, 1999, for a different account). It should be noted that in the counter model, prior study does not result in an increase in the probability of a diagnostic count ( $p s$ ). In signal detection terms, the counter model poses that repetition priming affects the criterion $\beta$, rather than the sensitivity $d^{\prime}$. The assumption that prior study does not increase sensitivity has been subject to debate (Bowers, 1999; Masson \& MacLeod, 1996; McKoon \& Ratcliff, in press).

As was mentioned earlier, repetition priming and frequency effects are mediated by different mechanisms in the counter model. In the counter model, word frequency affects the resting level of the counters. The resting level of counters of HF words is assumed to be higher than that of LF words. Therefore, when the alternatives consist of an HF word and an LF word, the counter of the HF word needs fewer additional counts to exceed the number of counts in the counter of the LF alternative by the criterion amount $k$ than vice versa. As a consequence, an HF target will be more often correctly identified than an LF target. Note, however, that performances for pairs consisting of two HF alternatives and for pairs consisting of two LF alternatives are predicted to be identical. This is because of the relative response criterion. The same number of additional counts has to be accumulated by the counter of the target in order to exceed the number of counts in the counter of the foil alternative by the criterion amount $k$, irrespective of whether both alternatives are either $\mathrm{HF}$ or LF words.

The counter model makes a series of parameterindependent and exact predictions (see the Appendix for a mathematical description of the counter model). First, prior study of both alternatives has no effect, because the counter model assumes that the attractive forces of the counters cancel each other out. Thus, performance should be equal for the conditions in which none of the alternatives is studied and for those conditions in which both alternatives are studied. Second, as was discussed above, no difference in performance is predicted between conditions with two HF alternatives and conditions with two LF alternatives, because of the relative response criterion. Only the difference in frequency between the two alternatives matters. This leads to the third prediction, that an $\mathrm{HF}$ alternative has an advantage when being paired with an LF alternative. Fourth, for pairs of alternatives consisting of an HF word and an LF word, bias effects (i.e., costs and benefits of prior study) are predicted to be larger if the LF word is the target than if the HF word is the target. Space limitations prevent us from presenting simulations confirming that the counter model makes this prediction. Intuitively, the LF counter needs relatively many counts to reach the criterion difference, and the more counts the decision process needs, the larger the impact of prior study becomes.

Although the counter model makes a number of nontrivial predictions on the relation between prior study and word frequency, these have not been systematically studied. In the present experiment, both word frequency (HF vs. LF) and study status (studied vs. nonstudied) of the target and the foil were independently varied. Thus, the target alternative could either be of the same frequency (either LF or HF) as the foil alternative or of different frequency, yielding a total of four target-foil frequencies (i.e., HF-HF, LF-LF, HF-LF, LF-HF; the first member of the pair denotes the frequency of the target, the second member denotes the frequency of the foil). In addition, there were four study conditions: Only the target was studied, only the foil was studied, both the target and the foil were studied, or neither the target nor the foil was studied.

\section{METHOD}

\section{Subjects}

Forty students at the University of Amsterdam participated for course credit. All the subjects were native speakers of Dutch and reported normal or corrected-to-normal vision.

\section{Design and Materials}

Word frequency and study status were varied independently in the present experiment. There were four frequency conditions. For half of the word pairs, the target and the foil were of equal frequency, either HF-HF or LF-LF. For the other half of the word pairs, the target and the foil were of unequal frequency, either HF-LF or LF-HF. For each frequency condition, there were four study conditions: Only the target was studied, only the foil was studied, both alternatives were studied, or neither alternative was 
Table 1

Proportion of Correctly Identified Targets as a Function of Word Frequency of the Target and the Foil and of Study Condition

\begin{tabular}{cccccc}
\hline & & \multicolumn{4}{c}{ Study Condition } \\
\cline { 2 - 5 } Target & Foil & Target & Foil & Neither & Both \\
\hline HF & LF & .870 & .773 & .868 & .842 \\
LF & HF & .842 & .683 & .757 & .793 \\
HF & HF & .862 & .753 & .816 & .822 \\
LF & LF & .874 & .720 & .765 & .821 \\
\hline
\end{tabular}

Note-HF, high-frequency word; LF, low-frequency word.

studied. Thus, the factor study condition had four levels, and in combination with the factor target-foil frequency of four levels, this resulted in a design of 16 experimental conditions.

The stimuli consisted of 384 similar word pairs (i.e., words of the same length differing in one letter, such as MOTHER-BOTHER) subdivided into three subsets. One subset consisted of 192 pairs of one $\mathrm{HF}$ alternative and one LF alternative. Another subset consisted of $96 \mathrm{HF}-\mathrm{HF}$ pairs, and a third subset consisted of $96 \mathrm{LF}-\mathrm{LF}$ pairs. Frequency counts were obtained from the CELEX norms (Baayen, Piepenbrock, \& van Rijn, 1993). The frequency of occurrence for all the HF words was higher than 30 per million (mean frequency, 231 per million). The frequency of occurrence for the LF words ranged between 1 and 5 per million (mean frequency, 2.6 per million). All the word stimuli were common Dutch words 4-7 letters long.

With the use of counterbalanced design, eight stimulus lists were created. Each list contained the same word pairs, but the study condition for each word pair was dependent on the list. Across the eight lists, each pair of alternatives consisting of an HF word and an LF word was rotated once through eight different conditions. In four conditions, the HF word was the target, and the LF word was the foil; in the other four conditions, the LF word was the target, and the HF word was the foil. Across the eight lists, both the HF-HF pairs and the LF-LF pairs were rotated twice through each of the four study conditions. Of each HF-HF pair and each LF-LF pair, one word was randomly assigned the target, and the other the foil. On each stimulus list, each word appeared only once. Each condition consisted of 24 trials. An additional 60 word pairs were used in the calibration phase in order to individually adjust the presentation time.

\section{Apparatus}

All the stimuli were presented on a Hewlett-Packard digital display module, model 1345A. The display module allowed stimulus presentation to be adjusted in steps of 2 msec. Stimulus presentation and response collection were controlled by an IBM personal computer.

\section{Procedure}

Our procedure closely followed the one used by Ratcliff and McKoon (1997). The instructions were a translated version of the instructions given by Ratcliff and McKoon in their experiments on perceptual forced choice. The important point of this instruction is that subjects were told to study words from the study blocks for a later, unspecified memory test (McKoon \& Ratcliff, in press).

The experimental stimuli were presented in 12 study-test blocks. Each study block consisted of 32 words and was followed by a test block consisting of 32 test trials. A 5-min break was given after the sixth test list. The order of the trials was randomly determined for each subject. During the study phase, the words were presented one at a time for $1 \mathrm{sec}$ per word. The transition from study list to test list was signaled by a warning sign consisting of a row of asterisks, displayed for $2.5 \mathrm{sec}$. Each test trial started with the presentation of a row of minus signs for $400 \mathrm{msec}$. Next, a blank screen was pre- sented for $300 \mathrm{msec}$. Subsequently, the test word was flashed. The flash time of the target was determined individually for each subject (see below). After presentation of the target, a 300-msec mask immediately covered the entire area where the test word had been presented. Immediately following the mask, two words were presented side by side on the line below. The subjects had to press the " $z$ " key with their left index finger if they thought the left-hand word was the flashed test word and the "?/" key with their right index finger if they thought the right-hand word was the flashed test word. Which was the correct choice, the right-hand alternative or the left-hand alternative, was chosen randomly. The mask consisted of eight mask characters. Ten types of pattern mask characters were used, each consisting of seven randomly oriented lines.

The first 4 trials were flashed for as long as $100 \mathrm{msec}$ in order to make sure that the requirements of the experiment were clear to the subject. Next, 60 calibration trials, subdivided into four blocks of 15 trials each, were presented, to estimate the flash time resulting in $70 \%$ correct performance. For this purpose, we used an adaptive algorithm in which the flash time in calibration block $N$ was adjusted on the basis of the performance in calibration block $\mathrm{N}-1$. Mean flash time for the subjects was $28.4 \mathrm{msec}$.

\section{RESULTS}

The proportions of correctly identified targets for the 16 conditions are presented in Table 1. Four analyses of variance (ANOVAs) were performed on the proportion of correctly identified targets, to focus on the specific predictions of the counter model. Two ANOVAs were performed on the results for the pairs of alternatives consisting of words of unequal frequency (i.e., HF-LF and LF-HF). One of these two ANOVAs was performed on the conditions in which either the target or the foil was previously studied. The other ANOVA was performed on the conditions in which either both the target and the foil were previously studied or neither the target nor the foil was previously studied. Likewise, two ANOVAs were performed on the results for the pairs of alternatives consisting of words of equal frequency (i.e., HF-HF and LF-LF).

The ANOVA for pairs of unequal frequency (HF-LF and LF-HF) of which either the target or the foil was studied showed a significant frequency effect $[F(1,39)=$ $\left.15.89, M S_{\mathrm{e}}=87.22, p<.001\right]$. HF targets were correctly identified more often than LF targets. The effect of study condition was also significant $\left[F(1,39)=67.86, M S_{\mathrm{e}}=\right.$ $95.97, p<.0001]$. Targets were correctly identified more often when the target was previously studied than when the foil was previously studied. The interaction between frequency of the target and study condition was also significant $\left[F(1,39)=7.38, M S_{\mathrm{e}}=51.20, p<.01\right]$. The effect of study condition was larger for LF targets than for $\mathrm{HF}$ targets.

The second ANOVA was performed for pairs of unequal frequency of which both the target and the foil were studied or neither the target nor the foil was studied. The frequency effect was significant $\left[F(1,39)=49.64, M S_{\mathrm{e}}=\right.$ $51.17, p<.0001]$. HF targets were correctly identified more often than LF targets. The effect of study condition was not significant $(F<1)$. However, the interaction be- 
tween frequency and study condition was significant $\left[F(1,39)=5.28, M S_{\mathrm{e}}=71.46, p<.05\right]$, indicating that prior study of both alternatives attenuated the word frequency effect.

The third ANOVA was performed for pairs of alternatives of equal frequency (HF-HF and LF-LF) of which either the target or the foil was studied. Performance did not differ between HF-HF pairs and LF-LF pairs $(F<1)$. There was a significant effect of study condition $[F(1,39)=$ $68.77, M S_{\mathrm{c}}=100.20, p<.0001 \mathrm{]}$, indicating that prior study of the target improved performance, as compared with prior study of the foil. The interaction between study condition and frequency condition was significant $\left[F(1,39)=5.25, M S_{\mathrm{e}}=40.03, p<.05\right]$, indicating that the effect of study condition was significantly larger for LF-LF pairs than for HF-HF pairs.

The fourth and last ANOVA was performed for pairs of equal frequency of which both the target and the foil were studied or neither the target nor the foil was studied. Performance for HF-HF pairs was better than that for LF-LF pairs $\left[F(1,39)=5.00, M S_{\mathrm{e}}=54.25, p<.05\right]$. In addition, performance was better when both alternatives were studied than when neither alternative was studied $\left[F(1,39)=5.97, M S_{\mathrm{e}}=65.43, p<.05\right]$. The interaction was again significant $\left[F(1,39)=4.42, M S_{\mathrm{e}}=56.58, p<\right.$ $.05]$. Simple main effects showed that the effect of frequency was significant when neither the target nor the foil was studied $\left[F(1,39)=8.10, M S_{\mathrm{e}}=64.32, p<.01\right]$, but not when both the target and the foil were studied $(F<1)$. Simple main effects also showed that effect of prior study was significant for LF targets $\left[F(1,39)=7.88, M S_{\mathrm{e}}=\right.$ $80.36, p<.01]$, but not for HF targets $(F<1)$.

\section{DISCUSSION}

In the present study, we replicated the findings of Ratcliff and McKoon (1997) that prior study of either the foil or the target leads to a bias effect. That is, prior study of the target leads to benefits, but prior study of the foil leads to costs. ' Moreover, in accordance with the counter model, this bias effect is more pronounced for LF targets paired with HF foils than for HF targets paired with LF foils. In contrast to the predictions of the counter model, however, we observed three interactions of word frequency with prior study. First, for pairs of equal frequency (HF-HF and LF-LF), the bias effect (i.e., the difference in proportion of correctly identified targets between conditions with prior study of the target and conditions with prior study of the foil) was larger for LF-LF pairs than for HF-HF pairs. This result raises problems for the counter model, because the model predicts identical performance for the LF-LF pairs and HF-HF pairs. For both types of word pairs, the resting level of the target equals that of the foil. Because of the relative response criterion, HF-HF conditions and LF-LF conditions are formally equivalent in the counter model. Second, for pairs of unequal frequency (HF-LF and LF-HF), prior study of both alternatives resulted in an attenuation of the word frequency effect of about $6 \%$. This interaction raises prob- lems for the counter model, because the model predicts that study of both alternatives has no effect whatsoever, as compared with the study of neither alternative. Third, performance for LF-LF pairs was worse than that for HF-HF pairs when neither alternative was studied. This difference in performance vanished when both alternatives were studied. As is the case for the former interaction, these findings raise problems for the counter model, because prior study of both alternatives should not affect performance. In addition, the counter model predicts that performance for HF-HF pairs is equivalent to that for LF-LF pairs. A final problem for the counter model is that prior study of an HF target did not lead to any improvement when the foil was an LF word (see Table 1). To summarize, the present findings indicate that (1) LF targets profit more from prior study than do HF targets even when both alternatives are of equal frequency, (2) prior study not only causes a bias effect but also causes an increase in discriminability or sensitivity, and (3) prior study of both alternatives attenuates the word frequency effect. These findings raise problems for the counter model.

As a possible objection against this conclusion, one might argue that the subjects used a strategy based on episodic memory for the studied items to help them reach a decision on some of the trials. Subjects might tend to choose an alternative because they explicitly recognize it as an item from the study list. In particular, because recognition is often found to be better for LF words than for HF words (see, e.g., Gregg, 1976), this could, perhaps, explain why prior study had a larger impact for LF targets than for HF targets. However, this explanation is not plausible for several reasons. First, study of an HF target did not result in any improvement in preference for it over a nonstudied LF foil. This is an improbable result if episodic retrieval strategies play an important role. Second, an explanation of the results in terms of episodic retrieval strategies cannot account for the observed increase in performance for LF-LF pairs when both alternatives were studied, as compared with that when none of the alternatives was studied. Third, we made sure that we used the same instructions as Ratcliff and McKoon (1997; McKoon \& Ratcliff, in press). McKoon and Ratcliff explicitly argue that their instruction leads subjects to refrain from using episodic strategies.

In the present study, using a procedure very similar to that of Ratcliff and McKoon (1997; McKoon \& Ratcliff, in press), we found that prior study of both alternatives attenuated the effect of word frequency. This replicates the traditional finding, obtained with free-response procedures, that effects of repetition priming are larger for LF words than for HF words (Jacoby \& Dallas, 1981). This result is predicted by many models, but not by the counter model. The relation between word frequency and repetition priming is of considerable theoretical importance. Many models of memory use one common mechanism to account for both phenomena. In PDP models, for instance, each new learning episode modifies connection weights between units, strengthening some and weakening others. In a PDP framework, an HF word is 
Table 2

Predictions of the Simple Linear Model for the Data From Table 1, With the Relevant Parameters Given in Parentheses

\begin{tabular}{ccllll} 
& & \multicolumn{4}{c}{ With } \\
\cline { 3 - 6 } Target & Foil & \multicolumn{2}{c}{ Target } & \multicolumn{3}{c}{ Foil } & Study Condition \\
\hline HF & LF & .888 & .786 & .837 & .837 \\
& & $\left(\mu+\delta+\beta_{\mathrm{s}}+\beta_{\mathrm{f}}\right)$ & $\left(\mu+\delta-\beta_{\mathrm{s}}+\beta_{\mathrm{f}}\right)$ & $\left(\mu+\delta+\beta_{\mathrm{f}}\right)$ & $\left(\mu+\delta+\beta_{\mathrm{f}}\right)$ \\
LF & HF & .845 & .695 & .746 & .794 \\
& & $\left(\mu+\delta+\beta_{\mathrm{s}}-\beta_{\mathrm{f}}\right)$ & $\left(\mu-\beta_{\mathrm{s}}-\beta_{\mathrm{f}}\right)$ & $\left(\mu-\beta_{\mathrm{f}}\right)$ & $\left(\mu+\delta-\beta_{\mathrm{f}}\right)$ \\
HF & HF & .866 & .764 & .815 & .815 \\
& & $\left(\mu+\delta+\beta_{\mathrm{s}}\right)$ & $\left(\mu+\delta-\beta_{\mathrm{s}}\right)$ & $(\mu+\delta)$ & $(\mu+\delta)$ \\
LF & LF & .866 & .717 & .768 & .815 \\
& & $\left(\mu+\delta+\beta_{\mathrm{s}}\right)$ & $\left(\mu-\beta_{\mathrm{s}}\right)$ & $(\mu)$ & $(\mu+\delta)$ \\
\hline
\end{tabular}

Note-HF, high-frequency word; LF, low-frequency word. Estimated values for the parameters are $\mu=.7677, \delta=.0476, \beta_{\mathrm{s}}=.0510, \beta_{\mathrm{f}}=.0216$.

simply a word that has received a lot of previous learning. It has been argued that such a learning mechanism makes the concept of frequency less ad hoc (Monsell, 1991). The counter model departs from these earlier models in that it posits two distinct mechanisms, one for word frequency and another for repetition priming. On a more theoretical level, this raises the question of how word frequency effects come about, if not through accumulated experience.

The other important finding of the present study is that for LF words, prior study results in enhanced discriminability and not just in bias. Of particular interest is the finding that in the LF-LF condition, prior study of both alternatives resulted in more correctly identified targets, as compared with prior study of neither alternative. This finding is difficult to explain by a mechanism that does not appeal, in one way or another, to some kind of facilitatory processing for repeated stimuli. A comparable result was recently obtained by Bowers (1999), who concluded that "priming can reflect a change in sensitivity rather than just bias, at least for low-frequency words." Below, we will present a descriptive quantitative model for our data and make a suggestion concerning the mechanism underlying enhanced discriminability.

A simple linear model is capable of quantitatively accounting for our results. The model makes four assumptions. The first assumption is that an HF target is identified better than an LF target, irrespective of the frequency of the foil. The second assumption is related to the first one and holds that an LF target is identified better after prior study, up to the point at which it is identified as well as an HF target. A psychological justification for these assumptions, albeit somewhat tentative, is that context availability is a factor involved in both word frequency and repetition priming. First, an HF item is seen in more contexts than an LF word prior to the forced-choice experiment and thus matches the test context more easily, leading to superior performance for HF items over LF items (cf. the first assumption). Second, prior study of an LF item will lead to a large increase in context availability for that item, whereas the increase in context availability for HF items will be relatively small, because HF items are already highly available in the test context (cf. the second assumption). The final two assumptions of the linear model deal with two bias effects: The study bias is the preference for a studied item over an unstudied item, and the frequency bias is the preference for an HF alternative over an LF alternative. The linear model requires four parameters. The first parameter, $\mu$, estimates baseline performance, in this case performance for unstudied LF-LF pairs. The second parameter, $\delta$, reflects a perceptual gain. This perceptual gain is inherent for HF items (see the first assumption) but can also be acquired by LF items through prior study (see the second assumption). The third and fourth parameters, $\beta_{\mathrm{s}}$ and $\beta_{\mathrm{f}}$, represent study bias and frequency bias, respectively (see the third and fourth assumptions). The parameters were estimated by using MINUIT (James \& Roos, 1975), a program that minimizes $\chi^{2}$, as a measure for the deviation between the data and the model. The model fits the data very nicely $\left[\chi^{2}(12)=14.26, p>.25\right.$; see Table 2 for details]. We do not propose this simple linear model as an alternative for process models, such as the counter model. Rather, the purpose is to highlight the implications of our data and to demonstrate that there is a simple set of assumptions by which to fully explain the observed pattern of results.

It remains to be seen whether modifications of the counter model are able to handle the data presented in this paper. A possible modification would be to let prior study as well as word frequency slightly enhance $p s$, the probability of perceiving a discriminating feature (as in the linear model described above). However, we believe that such an adjustment would be at odds with the core assumptions of the counter model. Furthermore, it is important to develop alternative models, such as the REM model (Schooler et al., 1999), to the point where they can be directly compared with the counter model. The counter model is currently the only model that tries to quantitatively predict effects of both word frequency and repetition priming in perceptual identification. We have presented evidence that these predictions of the counter model are false.

\section{SUMMARY AND CONCLUSIONS}

Three findings of the present study are of primary importance. First, the effects of prior study were larger for 
LF-LF pairs than for HF-HF pairs. This suggests that, contrary to the assumptions of the counter model, word frequency and repetition priming are mediated by a common mechanism. Second, for LF-LF pairs, performance was better when both alternatives were studied than when neither alternative was studied. This result indicates that, at least for LF words, prior study of words does not just result in bias. Rather, prior study also causes enhanced discriminability. Third, prior study of both alternatives attenuated the word frequency effect. This result is in line with traditional models of visual word recognition but is in direct conflict with the counter model.

\section{REFERENCES}

Baayen, R. H., Piepenbrock, R., \& van Rijn, H. (1993). The CELEX lexical database [CD-ROM]. University of Pennsylvania, Philadelphia: Linguistic Data Consortium.

BowERS, J. S. (1999). Priming is not all bias: Commentary on Ratcliff and McKoon (1997). Psychological Review, 106, 582-596.

FELLER, W. (1968). An introduction to probability theory and its applications. New York: Wiley.

GREGG, V. (1976). Word frequency, recognition and recall. In J. Brown (Ed.), Recall and recognition (pp. 183-216). London: Wiley.

JACOBY, L. L., \& DALLAS, M. (1981). On the relationship between autobiographical memory and perceptual learning. Journal of Experimental Psychology: General, 110, 306-340.

JAMES, F., \& Roos, M. (1975). MINUIT, a system for function minimization and analysis of the parameter errors and correlations. Computer Physics Communications, 10, 343-367.

Masson, M. E. J., \& MaCLeon, C. M. (1996). Contributions of processing fluency to repetition effects in masked word identification. Canadian Journal of Experimental Psvchology, 50, 9-21.

McClelland, J. L., \& RumelharT. D. E. (1981). An interactive activation model of context effects in letter perception: Pt. 1 . An account of basic findings. Psychological Review, 88, 375-407.

McKoon, G., \& Ratcliff, R. (in press). Bias in the counter model. Psvchological Review

MONSELL, S. (1991). The nature and locus of word frequency effects in reading. In D. Besner \& G. W. Humphreys (Eds.), Basic processes in reading: Visual word recognition ( $\mathrm{pp}$. 148-197). Hillsdale, NJ: Erlbaum.

MORTON, J. (1969). Interaction of information in word recognition. $P_{S v-}$ chological Review, 76, 165-178.

RatClifF, R., \& MCKoOn, G. (1997). A counter model for implicit priming in perceptual word identification. Psychological Review, 104, 319-343.

SchoOler, L., Shiffrin, R. M., \& RaAijmakers, J. G. W. (1999). The oretical note: A model for implicit effects in perceptual identification. Manuscript submitted for publication.

\section{NOTES}

1. When only the target is studied, the resulting increase in performance may be due to bias, as well as to an increase in sensitivity. In this particular condition, there appears to be no straightforward way to assess the relative contribution of these processes.

\section{APPENDIX \\ The Counter Model for Forced Choice: A Discrete Random Walk Process}

Mathematically, the counter model for perceptual forced choice (Ratcliff \& McKoon, 1997) is described by a discrete random walk process (e.g., Feller, 1968). The random walk model assumes that information is accumulated over time. At

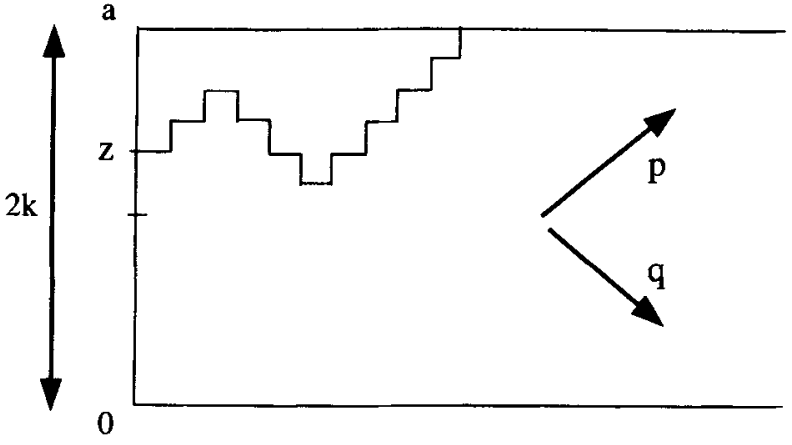

Figure A1. Illustration of the discrete random walk process. $Z$ is the location of the starting point; 0 and $a$ are the bottom and top boundaries, respectively; $p$ and $q$ are the probabilities of a step toward the top and bottom boundary, respectively; and $k$ is the critical difference in counts required to arrive at a decision. In the situation depicted in the figure, the stimulus is correctly identified (i.e., the top boundary is arrived at). Furthermore, the target alternative is a high-frequency word, and the foil is a lowfrequency word, which is evident from the displacement of the starting point $z$ toward the top boundary.

each time step, the random walk moves toward one of two boundaries. When the random walk hits a boundary, a decision is made.

For a discrete random walk process, the probability $q_{z}$ of absorption in the bottom boundary is given by

$$
q_{z}=\frac{(q / p)^{a}-(q / p)^{2}}{(q / p)^{a}-1},
$$

where $p$ is the probability of a step toward the upper boundary, $q$ is the probability of a step toward the lower boundary, $a$ is the location of the upper boundary, and $z$ is the location of the starting point. When a count is taken by one of the counters, the decision process takes one step toward the corresponding boundary (see Figure A1).

In the counter model, differences in frequency between the two alternatives affect the starting point of the random walk. When the choice is between an $\mathrm{HF}$ and an $\mathrm{LF}$ alternative, the starting point of the random walk is closer to the boundary of the HF word, and consequently, the HF word will need fewer counts than the LF word to hit the boundary. For two HF alternatives, as well as for two LF alternatives, the distance from the starting point to either boundary is identical (i.e., $k$ ). The counter model, therefore, predicts that performance for the HF-HF condition should be identical to that for the LF-LF condition.

Unlike word frequency, prior study does not affect the starting point but does affect the probability of a step toward a boundary. When the choice is between a studied and a nonstudied alternative, the probability of a step toward the boundary of the studied alternative is slightly increased, and consequently, the probability of a step toward the boundary of the nonstudied alternative is slightly decreased, as compared with those cases in which neither alternative is studied or both alternatives are studied.

(Manuscript received May 4, 1999 revision accepted for publication September 17,1999 .) 